\title{
All-optical OFDM and Distributed Raman Amplification: challenges to enable high capacities and extend reach
}

\author{
Fatima C. Garcia Gunning ${ }^{1}$, Member, IEEE, Paola Frascella ${ }^{1, *}$, Student Member, IEEE, Cleitus Antony ${ }^{1}$, \\ Member, IEEE, Simon J. Fabbri ${ }^{1}$, Paul Gunning ${ }^{2}$, William McAuliffe ${ }^{3}$, Derek Cassidy ${ }^{3}$, \\ and Andrew D. Ellis ${ }^{1}$ \\ ${ }^{1}$ Photonic Systems Group, Dept. Physics and Tyndall National Institute, University College Cork, Ireland \\ ${ }^{2}$ BT Innovate and Design, Adastral Park, Martlesham Heath, Ipswich, IP5 3RE, U.K. \\ ${ }^{3}$ BT Ireland, Grand Canal Plaza, Upper Grand Canal St., Dublin 4, Ireland \\ Tel: +353 (21) 490 4859, Fax: +353 (21) 490 4880, e-mail: fatima.gunning@tyndall.ie
}

\begin{abstract}
We review recent advances in all-optical OFDM technologies and discuss the performance of a field trial of a 2Tbit/s Coherent WDM over $124 \mathrm{~km}$ with distributed Raman amplification. The results indicate that careful optimisation of the Raman pumps is essential. We also consider how all-optical OFDM systems perform favourably against energy consumption when compared with alternative coherent detection schemes. We argue that, in an energy constrained high-capacity transmission system, direct detected all-optical OFDM with 'ideal' Raman amplification is an attractive candidate for metro area datacentre interconnects with $\sim 100 \mathrm{~km}$ fibre spans, with an overall energy requirement at least three times lower than coherent detection techniques.
\end{abstract}

Keywords: Raman amplification, all-optical OFDM, energy consumption.

\section{INTRODUCTION}

The demand for transmission services that consume appreciable optical bandwidth capacity, such as those required to interconnect warehouse scale computing facilities ${ }^{1}$, continues to increase and, if ignored, could adversely impact the supply of bandwidth available from within the optical plant that has been installed by network operators, who are now faced with declining revenues per carried bit. Several mitigating solutions have been suggested, including the manipulation of the full optical field variables, such as amplitude, phase, time, frequency and polarization, and, more recently, spatial multiplexing techniques, either with multi-mode multiplexing or multi-core transmissions, are also being investigated. Technologies that replace current installed optical fibres are also being discussed, with waveguide geometries that allow larger effective areas to minimise nonlinearities and/or enable few-mode propagation, or multiple cores to increase the throughput in a single cable, or even novel optical fibres, such as hollow-core microstructure fibres, to enable very low loss transmission in addition to higher power handling. The more pragmatic near-term approach for a Network operator to follow would exploit all the possible bandwidth available from within its deployed ITU-T standard (G.652) ${ }^{2}$ optical fibre plant to arrest margin erosion. It is therefore more cost-effective to upgrade the terminal equipment (transmitters and receivers) attached to each end of the deployed transmission plant. This encourages the development of terminal equipment that exploits all the optical field variables for information encoding first and so extend the operational lifetime of the deployed optical transmission plant.

With this in mind, recently proposed techniques such as optical OFDM ${ }^{3}$, higher order modulation formats ${ }^{4}$, and polarisation multiplexing ${ }^{5}$, whether implemented individually or in combination ${ }^{6}$, have been amongst the terminal techniques adopted to extend the operational lifetime of installed optical fibre plant. These techniques can be further usefully augmented with extended C-band and Distributed Raman amplifiers (DRA.) However, less appreciated, but no less significant, is the energy consumed to support these techniques which impacts the initial capital outlay and the ongoing operational cost for the network operator. For example, DRA requires high pump powers, increasing the costs of implementation and maintenance, while coherently detected higher order modulation formats (e.g. 128QAM) require high performance lasers and power-hungry high speed digital signal processing (DSP) circuits. In this paper we will show some indications that the combination of DRA and directly detected all-optical OFDM (or Coherent WDM) offers an attractive energy consumption even for high capacities and long reach systems. We will report the impact of using DRA with a 2Tbit/s all-optical OFDM on a fieldinstalled optical transmission link of $124 \mathrm{~km}$ length.

\section{IMPLEMENTATION OF SUPERCHANNELS}

The demonstration of all-optical OFDM in single mode fibre was first seen in $2002^{7}$, where the concepts from wireless communications were transferred to the optical domain with the aid of an optical discrete Fourier transformer based on asymmetric Mach-Zehnder interferometers. This work was followed by the experimental

\footnotetext{
${ }^{*}$ now with Nokia Siemens Networks Portugal, S.A., Lisbon, Portugal
} 
demonstration of Coherent $\mathrm{WDM}^{8}$, where it was shown that, even without ideal matched filters, the phase between adjacent subcarriers at the transmitter could be carefully adjusted to preserve orthogonality. Since then similar concepts have been introduced, for example: coherent $\mathrm{OFDM}^{3}$, taking advantage of coherent detection, and hence DSP which enables the use of matched filters; and the label 'superchannel'" has been introduced for systems based on these concepts achieving very high capacities and spectral efficiencies. Superchannels have attracted a lot of industrial attention ${ }^{10,11}$, because they allow solutions beyond the $100 \mathrm{Gbit} / \mathrm{s}$ state-of-the-art, whether it being $400 \mathrm{Gbit} / \mathrm{s}$ or $1 \mathrm{Tbit} / \mathrm{s}^{12,13}$. Advantages of using superchannels include the high capacity throughput and inherit high spectral efficiency, while restricting the impact of transmission degradations from impairments such as PMD and nonlinearity, to those typically encountered at the baud rate of the constituent subcarriers.

A typical transmitter for a direct detected all-optical OFDM system is illustrated in Figure 1. In this case, the laser bank was composed of seven DFB lasers, separated by $385.56 \mathrm{GHz}$, which were passively combined and the resultant signal amplified to $23 \mathrm{dBm}$ before launching to the comb generator. The comb generator has been explained elsewhere ${ }^{14}$, and it was composed of two cascaded Mach-Zehnder modulators (MZM) driven by a clock signal at $42.84 \mathrm{GHz}$, generating seven bands of seven subcarriers, each of which was separated by 42.84GHz. A small dispersion compensating fibre (DCF) was added to equalise the relative phases of the subcarriers after the second EDFA $(27 \mathrm{dBm})$ prior to data encoding. A disinterleaver was used to separate odd and even channels, which were independently data encoded at 42.84Gbaud with decorrelated pseudo-random sequences at $2^{31}-1$ and non-return-to-zero on-off keying (NRZ-OOK) format, although differential phase shift keying (DPSK) has also been demonstrated. Polarisation controllers (PC) ensured that all 49 subcarriers were copolarised prior to transmission (at point A, in Figure 1a), with a total power of $8.7 \mathrm{dBm}$ over $2.8 \mathrm{THz}$ bandwidth, transporting a total of 2.1Tbit/s. A phase stabilisation feedback loop based on the RF beat note between adjacent subcarriers ensured that the relative phases between subcarriers were optimised.
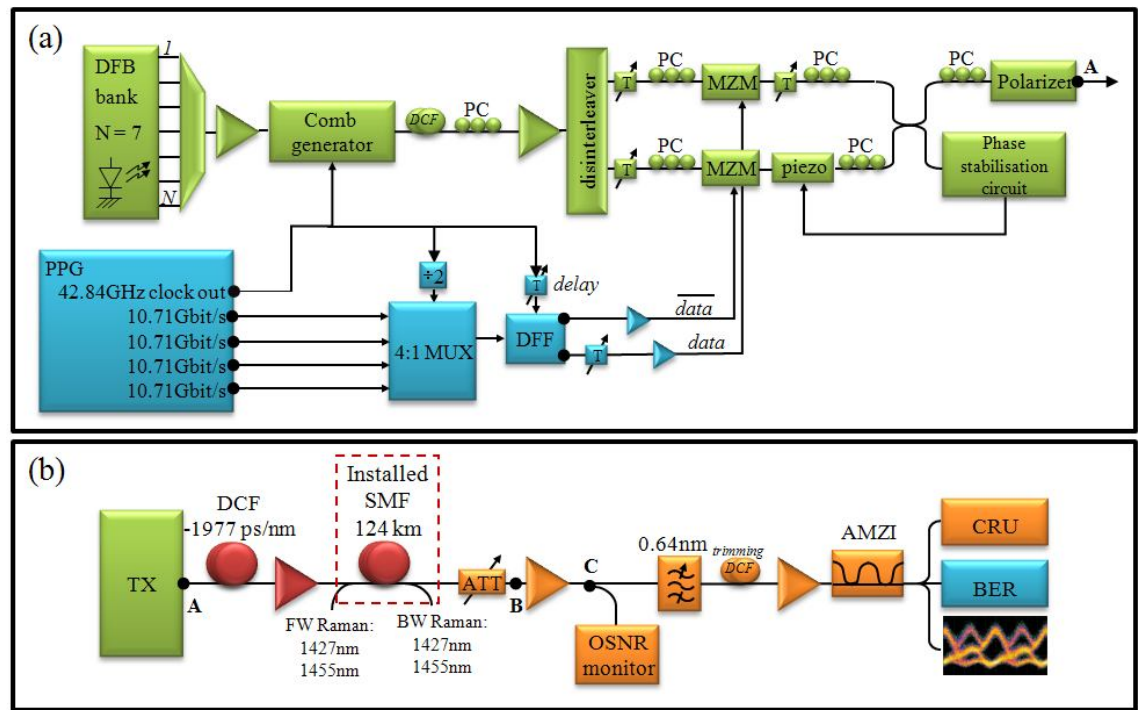

Figure 1: Schematic of a 49 subcarriers Coherent WDM transmitter (a), transmitting over 124km of installed SMF fibre, and direct detected by the receiver (b). PCs stand for polarisation controllers and $T$ for delay lines.

The co-polarised signal at point A in Figure 1(a) and Figure 1(b) was then pre-compensated with a dispersion compensating fibre (DCF), totalling $-1977 \mathrm{ps} / \mathrm{nm}$, with $9 \mathrm{~dB}$ loss and a nonlinear coefficient of $\sim 7.6 \mathrm{~W}^{-1} \mathrm{~km}^{-1}$, prior to the link EDFA (red triangle, Figure 1(b)). Transmission over 124km of BT-Ireland's installed single mode fibre (SMF) between Cork and Clonakilty (and looped back to Cork) occurred either with backward (BW) or forward (FW) Raman amplification, which included an in-band and out-of-band supervisory channels ${ }^{15}$. DRA was provided by two pumps each (at 1427 and $1455 \mathrm{~nm}$ ) at a maximum power of $+27 \mathrm{dBm}$. This scheme was implemented to optimise the optical signal-to-noise ratio (OSNR) at the receiver, and was optimised to balance the OSNR benefits with penalties associated with the peak-to-average power ratio increase. The direct detection receiver, as in Figure 1(b), was also described previously. An attenuator (ATT) followed by a power monitor, measuring the total input power to the receiver at point $\mathrm{B}$, was added between the fibre output and the receiver input to enable system characterisation. A pre-amplified receiver was used, where the OSNR from the small signal gain EDFA was monitored with an optical spectrum analyser. The OSNR measured was defined as the ratio of the signal power in a band $(2.5 \mathrm{~nm})$ over the noise power in $0.1 \mathrm{~nm}$. In order to select each individual subcarrier for data analysis, a combination of a $0.64 \mathrm{~nm}$ tuneable bandpass filter and an asymmetric Mach- 
Zehnder interferometer (AMZI) of $85.66 \mathrm{GHz}$ free spectral range was used (also known as a one tap discrete Fourier transform ${ }^{13}$ ). The receiver also contained DCF to compensate for any residual dispersion from the link.

\section{DISTRIBUTED RAMAN AMPLIFICATION (DRA)}

Optimisation of a DRA system is complex because the distribution of the gain along the transmission fibre reduces the total ASE and improves OSNR, but simultaneously increases the signal distortions that result from Kerr and other nonlinearities. The nonlinear response of the system was analysed by varying the signal launch power, or the link-EDFA gain $\left(\mathrm{G}_{\mathrm{EDFA}}\right)$, and measuring the transmission performance of the $2 \mathrm{Tbit} / \mathrm{s}$ data signal. In this case, a fixed BW Raman gain was fixed to 17.2dB. Both OSNR at the output of the link-EDFA and the total BER after transmission were measured. Figure 2(a) shows the equivalent calculated Q-factor from the real-time BER measurements for subcarrier \#48, together with the OSNR, when varying $\mathrm{G}_{\mathrm{EDFA}}$ using the automatic gain control (AGC) feature and taking into account additional losses from the monitoring taps. Clearly an optimum Q-factor was observed when the power per subcarrier was $\sim-1 \mathrm{dBm}$ at the input of the installed link, or a $\mathrm{G}_{\mathrm{EDFA}}$ of $17.7 \mathrm{~dB}$, with an input OSNR to the link of $\sim 36.7 \mathrm{~dB}$. At low power levels the Q-factor is degraded by a reduced OSNR, and, at high powers, by nonlinearities, as expected.
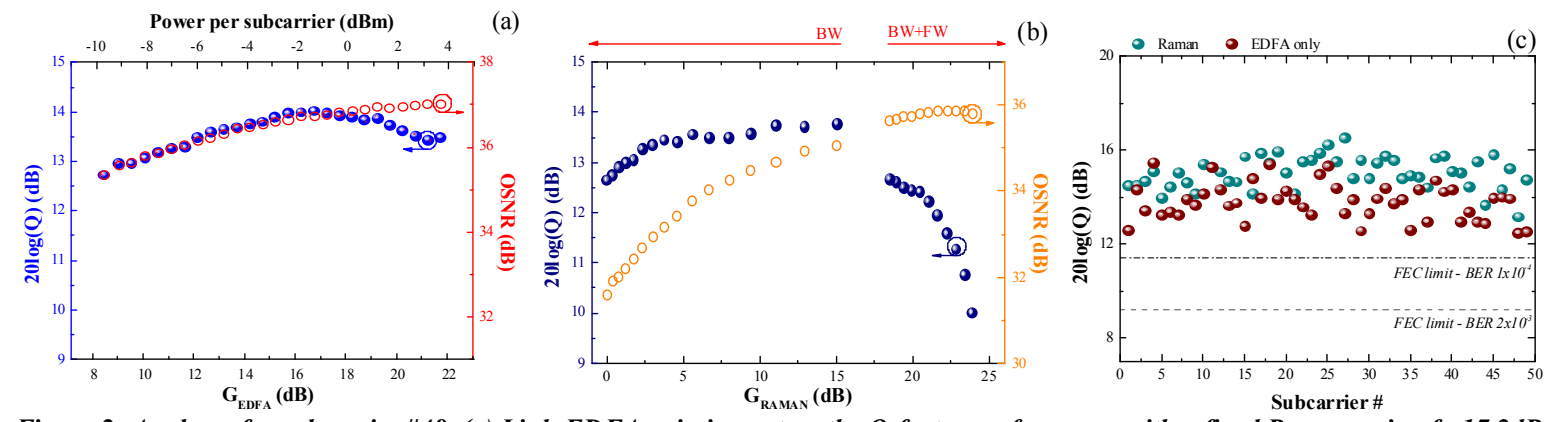

Figure 2: Analyses for subcarrier \#48. (a) Link-EDFA gain impact on the O-factor performance with a fixed Raman gain of $\sim 17.2 d B$. OSNR measured at the output of the link-EDFA. (b) Raman gain impact on Q-factor performance for both backward and backward+forward Raman, with a fixed $G_{E D F A}$ of $17.7 \mathrm{~dB}$. OSNR in this case was measured at the output of the receiver pre-amplifier. (c) Overall Q-factor transmission performance over $124 \mathrm{~km}$ of installed fibre for all 49 subcarriers with EDFA-only amplification, and combined EDFA and BW Raman only.

For the Raman amplification system, two configurations were studied, with $\mathrm{G}_{\mathrm{EDFA}}$ set to $17.7 \mathrm{~dB}$. For low overall Raman gains only the BW Raman pumps were switched on. Pumps for both wavelengths were set to the same power levels at all times. For higher Raman gains, the backwards pumps were operated at their maximum output power, and the FW pump powers (equal power at both pump wavelengths) were set to give the required Raman gain. Figure 2(b) shows the results (also for subcarrier \#48), where the Q-factor and OSNR at the receiver (point $\mathrm{C}$ in Figure 1) are shown against the change in Raman gain $\left(\mathrm{G}_{\mathrm{RAMAN}}\right)$. For low gains (BW only) the OSNR increased steadily, as expected. However, the Q-factor saturates once a gain of $\sim 5 \mathrm{~dB}$ is reached, giving a maximum improvement of $1.2 \mathrm{~dB}$. For higher gains (including FW DRA) the performance degrades rapidly. This occurs due to an increase in nonlinearities as the FW pumps increase both the overall launch power to the fibre and the effective length. We believe that the balance between signal launch powers and DRA gain in this experiment was close to the optimum configuration for a $124 \mathrm{~km}$ span for a first order DRA, and the results were compared to an optimised EDFA only system (as in $\left[{ }^{16}\right]$ ), as shown in Figure 2(c). Again, it clearly shows an average improvement of $1.2 \mathrm{~dB}$ in Q-factor, well above the FEC thresholds.

\section{DISCUSSIONS ON ENERGY CONSUMPTION}

As suggested earlier, network operators face the challenge of meeting the current demands for optical bandwidth capacity, whilst extending the operational lifetime of the deployed optical transmission plant. Here we presented possible solutions by implementing direct detected all-optical OFDM with the aid of DRA, although solutions with higher order modulation formats have also been demonstrated elsewhere. Such formats, such as m-QAM, are likely to be adopted in the near future to allow high capacities and extended reaches, with maximum reach achieved with coherent detection, and hence, at the expense of much higher energy consumption. In order to analyse this trade off, we calculated the total link energy consumption as follows. Firstly, the required signal launch power for a given reach and net information spectral density (ISD) was calculated according to the conventional capacity limit equations ${ }^{17}$, including an approximation for the reduction in the system capacity when using direct detection receivers ${ }^{18}$, and assuming that ideal lossless Raman amplification may be used ${ }^{19}$ [Rene, Aston]. We then calculated the required total optical pump power for the system (summed over all amplifiers), assuming transparent operation for the DRA system $\left(1^{\text {st }}\right.$ order pumping), or $1480 \mathrm{~nm}$ pumped EDFAs otherwise, and multiplied by a constant factor which reflects the energy efficiency of 
commercially available devices ${ }^{20}$. We added to this the energy consumption of the transponders ${ }^{21}$, and, for the coherent detection case, we assumed optimistically that higher order modulation formats may be implemented without any significant increase in energy consumption. In Figure 3(a), we show that, for a system comprising $28 \mathrm{Gbaud}$ WDM transponders, covering $2 \mathrm{THz}$ total bandwidth with $50 \mathrm{GHz}$ channel spacing, 100km EDFA spacing and digital coherent detection, total energy consumption levels of the link exceeds $16 \mathrm{~kW}$ irrespective of reach and distance. This is due to the energy consumption of the DSP circuitry of around $8 \mathrm{~W} / \mathrm{GHz}$ of bandwidth. Conversely, as shown in Figure 3(b), when orthogonal subcarriers with optical matched filters and direct detection (1.4 W/GHz power consumption) are used to increase the spectral efficiency, and DRA is employed to increase the reach, the energy requirements are decreased by almost six times for short reach systems and by more than a factor of two for long haul systems and are dominated by the required signal launch power, rather than the transponders'. The graphs presented here indicate that, while for longer reaches an increase in energy use may be justified, for shorter reaches, where the costs are constrained, the direct detected orthogonal techniques may be more advantageous. Moreover, there is a clear trade-off between reach and ISD, as expected, due to the struggle between increasing the launch powers and minimising the nonlinear impairments.
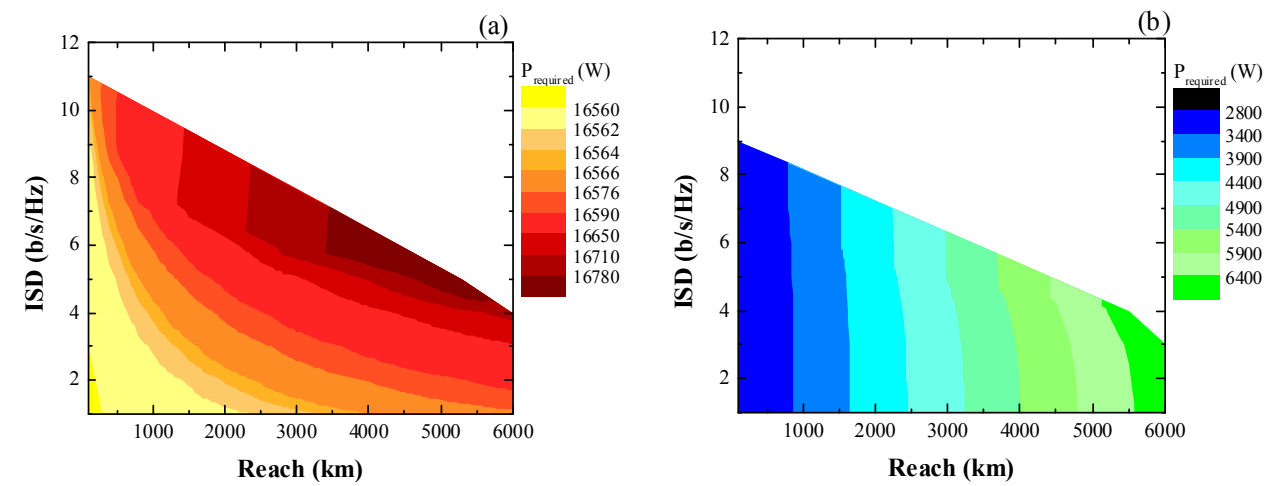

Figure 3: Contour plots of total required power $\left(P_{\text {required }}\right)$ against reach and information spectral density (ISD) for a 2THz bandwidth signal using (b) a standard WDM 28Gbaud transponder, separated by 50GHz, using coherent detection; and (b) a direct-detected alloptical OFDM transponder, with 28 Gbaud, separated by $28 \mathrm{GHz}$, using ideal Raman amplification. In all cases, the amplifier spacing was set to $100 \mathrm{~km}$ of SMF (dispersion of $20 \mathrm{ps} / \mathrm{nm} / \mathrm{km}$ and nonlinear coefficient of $1.4 / W / \mathrm{km}$.) White regions indicate that error free transmission is not possible.

\section{CONCLUSIONS}

In this paper we have demonstrated how direct detected orthogonal techniques, such as all-optical OFDM, may play a much bigger role in the near future due to its lower energy requirements, when compared to coherent detected techniques, unless the digital signal processing energy consumption is reduced by almost an order of magnitude. We demonstrated an overall 1.2dB Q-factor improvement for 2Tbit/s direct detected all-optical OFDM when using an optimised DRA over a $124 \mathrm{~km}$ installed link.

\section{ACKNOWLEDGEMENTS}

This work was supported by Science Foundation Ireland under Grant 06/IN/I969, 07/SRC/I1173, and 10/CE/I1853.

\section{REFERENCES}

[1] L.A. Barroso and U. Hölzle: The Datacenter as a Computer: An Introduction to the Design of WarehouseScale Machine, in Synthesis Lectures on Computer Architecture, M.D. Hill, Ed., Morgan \& Claypool Publishers (2009).

[2] ITU-T Recommendation G.652: Characteristics of a single mode optical fibre and cable

[3] W. Shieh and C. Athaudage: Coherent optical orthogonal frequency division multiplexing, in Elect. Lett., vol. 42, n. 10, pp. $587-589$, May 2006.

[4] H. Goto, et al: Polarization-multiplexed $1 \mathrm{Gsymbol} / \mathrm{s}, 128 \mathrm{QAM}(14 \mathrm{Gbit} / \mathrm{s})$ coherent optical transmission over $160 \mathrm{~km}$ using a $1.4 \mathrm{GHz}$ Nyquist filter, in Proc. OFC/NFOEC 2008, San Diego, USA, Feb. 2008, paper JThA45.

[5] S. Bhandare, et al: 5.94-Tb/s 1.49-b/s/Hz (40 $2240 \mathrm{~Gb} / \mathrm{s})$ RZ-DQPSK Polarization-Division Multiplex C-Band Transmission Over 324 km, in IEEE Phot. Tech. Lett., vol. 17, n. 4, pp. 914-916, April 2005.

[6] A. Sano, et al: Ultra-high Capacity Optical Transmission Technologies for 100Tbit/s Optical Transport Networks, IEICE Trans. Commun., vol. E94-B, n.2, pp. 400-408, Feb. 2011. 
[7] H. Sanjoh, et al: Optical Orthogonal Frequency Division Multiplexing using Frequency/Time Domain Filtering for High Spectral Efficiency up to $1 \mathrm{bit} / \mathrm{s} / \mathrm{Hz}$, in Proc. OFC 2002, paper ThD1.

[8] F.C.Garcia Gunning, et al: $298 \mathrm{Gbit} / \mathrm{s}$ Coherent WDM Transmission over $80 \mathrm{~km}$ of SMF at $1 \mathrm{bit} / \mathrm{s} / \mathrm{Hz}$ Spectral Efficiency, in Proc. ECOC 2005, Glasgow, UK, paper We3.2.6.

[9] S. Chandrasekhar, et al: Transmission of a $1.2-\mathrm{Tb} / \mathrm{s}$ 24-carrier no-guard-interval coherent OFDM superchannel over 7200-km of ultra-large area fiber, in Proc. ECOC 2009, Vienna, Austria, Sept. 2009, paper PD2.6.

[10] Infinera whitepaper: Super-Channels DWDM Transmission Beyond 100Gb/s: Will Bandwidth Growth Ever Stop? No. http://www.infinera.com/pdfs/whitepapers/SuperChannel WhitePaper.pdf

[11] NEC news room: NEC Demonstrates First Terabit/s Superchannel Transmission over 10,000km, January 2012. http://www.nec.co.jp/press/en/1201/1001.html

[12] X. Liu, et al: $1.12-\mathrm{Tb} / \mathrm{s}$ 32-QAM-OFDM superchannel with $8.6-\mathrm{b} / \mathrm{s} / \mathrm{Hz}$ intrachannel spectral efficiency and space-division multiplexed transmission with $60-\mathrm{b} / \mathrm{s} / \mathrm{Hz}$ aggregate spectral efficiency, in Opt. Exp., vol. 19, n. 26, pp. B958-B964, December 2011.

[13] D. Hillerkuss, et al.: 26Tbit/s line-rate super-channel transmission utilizing all-optical fast Fourier Transform Processing, in Nature Phot., vol. 5, pp. 364-371, May 2011.

[14] T. Healy, et al: Multi-Wavelength Source using Low Drive Voltage Amplitude Modulators for High Information Spectral Density Systems, in Opt. Exp., vol. 15, n. 6, pp. 2981-2986, 2007.

[15] P. Frascella, et al: Impact of Raman Amplification on a 2-Tb/s Coherent WDM System, in Phot. Tech. Lett., vol. 23, n. 14, pp. $959-961,2011$.

[16] P. Frascella, et al.: Unrepeatered field transmission of $2 \mathrm{Tbit} / \mathrm{s}$ multi-banded coherent WDM over $124 \mathrm{~km}$ of installed SMF, in Opt. Exp., vol. 18, n. 24, pp. 24745-24752, Nov. 2010.

[17] P. P. Mitra and J. B. Stark: Nonlinear limits to the information capacity of optical fibre communications, in Nature, vol. 411, pp. 1027, 2001

[18] J. M. Kahn and K.-P. Ho: Spectral efficiency limits and modulation/detection techniques for DWDM systems, in J. Select. Topics Quantum Electron., vol. 10, n. 2, pp. 259-272, Mar./Apr. 2004.

[19] R.J. Essiambre, et at: Capacity Limits of Information Transport in Fiber-Optic Networks, in Phys. Rev. Lett. vol. 101, pp. 163901, Oct. 2008.

[20] W.V. Heddeghem et al: Power consumption modeling in optical multilayer networks, in Phot. Net. Comm., Jan. 2012.

[21] A. Morea, et al: Power Management of Optoelectronic Interfaces for Dynamic Optical Networks, in Proc. ECOC 2011, paper We.8.K.3. 